\title{
Pathway deviation-based biomarker and multi-effect target identification in asbestos-related squamous cell carcinoma of the lung
}

\author{
JIANG DU and LIN ZHANG \\ Department of Thoracic Surgery, First Affiliated Hospital of China Medical University, Shenyang, Liaoning 110001, P.R. China
}

Received March 3, 2016; Accepted January 20, 2017

DOI: $10.3892 / \mathrm{ijmm} .2017 .2878$

\begin{abstract}
Asbestos-related lung carcinoma is one of the most devastating occupational cancers, and effective techniques for early diagnosis are still lacking. In the present study, a systematic approach was applied to detect a potential biomarker for asbestos-related lung cancer (ARLC); in particular asbestos-related squamous cell carcinoma (ARLC-SCC). Microarray data (GSE23822) were retrieved from the Gene Expression Omnibus database, including 26 ARLC-SCCs and 30 non-asbestos-related squamous cell lung carcinomas (NARLC-SCCs). Differentially expressed genes (DEGs) were identified by the limma package, and then a protein-protein interaction (PPI) network was constructed according to the BioGRID and HPRD databases. A novel scoring approach integrating an expression deviation score and network degree of the gene was then proposed to weight the DEGs. Subsequently, the important genes were uploaded to DAVID for pathway enrichment analysis. Pathway correlation analysis was carried out using Spearman's rank correlation coefficient of the pathscore. In total, 1,333 DEGs, 391 upregulated and 942 downregulated, were obtained between the ARLC-SCCs and NARLC-SCCs. A total of 524 important genes for ARLC-SCC were significantly enriched in 22 KEGG pathways. Correlation analysis of these pathways showed that the pathway of SNARE interactions in vesicular transport was significantly correlated with 12 other pathways. Additionally, obvious correlations were found between multiple pathways by sharing cross-talk genes (EGFR, PRKX, PDGFB, PIK3R3, SLK, IGF1, CDC42 and PRKCA). On the whole, our data demonstrate that 8 cross-talk genes were found to bridge multiple ARLC-SCC-specific pathways, which may be used as candidate biomarkers and potential multi-effect
\end{abstract}

Correspondence to: Dr Lin Zhang, Department of Thoracic Surgery, First Affiliated Hospital of China Medical University, 155 Nanjingbei Street, Heping, Shenyang, Liaoning 110001, P.R. China

E-mail: linzhangdoc@163.com

Key words: asbestos-related lung cancer, biomarker, gene microarray, network analysis, squamous cell carcinoma targets. As these genes are involved in multiple pathways, it is possible that drugs targeting these genes may thus be able to influence multiple pathways simultaneously.

\section{Introduction}

Lung cancer has continued to be the most common type of cancer worldwide for several decades, with the highest incidence and mortality rates (1). Only $13 \%$ of patients with lung cancer survive for $>5$ years (2). As the second leading risk factor for lung cancer, asbestos exposure is responsible for an estimated 5-7\% of all these cancers (3). Asbestos-related lung carcinoma is considered to be one of the most devastating occupational cancers (4). Although the use of asbestos has been banned or severely restricted since the early 1970's in many developed countries, asbestos-related lung cancer still poses a great public health threat due to the long latency period from asbestos exposure to the incidence of asbestos-induced cancer (5).

It has been widely accepted that early detection and precise diagnosis of cancer subtypes could greatly enhance the efficacy of targeted therapies and improve disease outcome, and in fact, markedly increase the patient survival rate (6). To date, many imaging and cytology-based methods have been applied for early detection (7-9). However, most techniques have limited sensitivity to detect asbestos-related lung cancer, as the histopathological subtypes of lung cancer patients with and without asbestos exposure are quite similar (10).

Among asbestos-related lung cancer, non-small cell lung cancer (NSCLC) accounts for at least $80 \%$ of these cases (2). There are three primary subtypes of NSCLC distinguishable by the appearance and chemical makeup of the cells: adenocarcinoma (LC-AC), squamous cell carcinoma (LC-SCC) and large-cell carcinoma. It has been shown that gene expression profiles could be used to distinguish asbestos-exposed from non-exposed lung cancer patients (11). Gene expression profiles in asbestos-exposed epithelial and mesothelial lung cell lines have revealed that the expression levels of genes such as nuclear factor- $\kappa \mathrm{B}(\mathrm{NF}-\kappa \mathrm{B})$ subunit 2 (NFKB2), IKBKB, thioredoxin (TXN), thioredoxin reductase (TXNRD1), BCL2 interacting protein 3 like (BNIP3L), protein kinase $\mathrm{C}(\mathrm{PKC}) \delta$ and adducin (ADD) 3 were significantly altered in response to asbestos exposure in all the cell lines (12). Moreover, accumulating in vivo and in vitro studies have identified asbestos-related 
gene expression changes involved in activation of the $\mathrm{NF}-\kappa \mathrm{B}$ pathway, p53 promoter activation, MAPK signaling pathway and cell proliferation induced by tumor necrosis factor- $\alpha$ (TNF- $\alpha$ ) and TNF- $\beta$ as well as PDGFA and PDGFB $(13,14)$. The increase in available tumor samples of patients diagnosed with different subtypes of a certain tumor makes it possible to detect subtypespecific biomarkers. Class comparison analysis of tumors of 36 patients with primary LC-AC identified ADAM28 as a potential oncogene involved in asbestos-related LC-AC, with expression verified in three independent test sets (15). Similarly, gene expression profiling of asbestos-related lung squamous cell carcinomas (ARLC-SCCs) identified MS4A1 as a potential candidate (10). However, immunohistochemical staining showed that expression of MS4A1 was primarily localized to stromal lymphocytes rather than tumor cells. Thus, identification of candidate biomarkers in ARLC-SCC is needed.

Using the same data from Wright et al (10), we aimed to further identify differentially expressed genes (DEGs) according to a cut-off point of a $\log _{2}$ fold-change $(\mathrm{FC})>1$ or $<-1$ and $\mathrm{P}$-value $<0.05$. Furthermore, the protein-protein interaction (PPI) network was constructed and a novel, pathway-deviation-based approach was proposed to detect potential ARLC-SCC-specific biomarkers from the networks. Notably, a new scoring method was developed to weight each DEG by integrating its expression deviation score and network degree. In addition, a newly defined parameter, pathscore, was used to measure the deviation in each pathway enriched by DEGs. Finally, the molecular pathways involved in the disease development of these two types of lung cancer samples and their molecular heterogeneity, were systematically analyzed by hierarchical clustering and pathway correlation analysis. Several candidate marker genes and multieffect targets were identified, which may provide insight into the development of novel diagnostic and therapeutic tools for ARLC-SCC. In addition, as these genes are involved in multiple pathaws, they may thus function as 'multi-effect targets', as it is possible, that drugs targeting these genes may influence multiple pathways simultaneously.

\section{Materials and methods}

Gene differential expression analysis. The microarray data were retrieved from Gene Expression Omnibus (GEO; https:// www.ncbi.nlm.nih.gov/geo/) database under accession no. GSE23822 (10). Total RNAs from 56 human lung squamous cell carcinoma samples, consisting of 26 ARLC-SCCs and 30 non-asbestos-related squamous cell lung carcinomas (NARLC-SCCs), were hybridized to $48 \mathrm{~K}$ Illumina HumanHT-12 v3.0 Expression BeadChips, respectively. These cases were classified as ARLC-SCC if there were $>20$ asbestos bodies/gram wet weight (AB/g ww) in the non-tumor tissue, or NARLC-SCC if no asbestos bodies were found. No statistically significant differences in age, gender, smoking history or tumor stage was noted between the two types of samples. After background correction, the intensities of multiple probes for a gene were averaged into one expression value which was then transformed as a normalized expression value by $\mathrm{Z}$-score. The differential expression analysis between the two groups of samples was performed using the R package known as linear models for microarray data (LIMMA) (16). To minimize the potential information loss caused by multi-hypothesis test and reserve more inter-group DEGs, a P-value before false discovery rate (FDR) adjustment was used to detect the significant DEGs and the detailed criterion of DEGs was defined as $\mathrm{P}<0.05$.

Protein-protein interaction (PPI) network construction. PPI data were downloaded from the Biological General Repository for Interaction Datasets (BioGRID; http://www.thebiogrid. org) interaction database (17) and Human Protein Reference Database (HPRD; http://www.hprd.org) (18), and merged into the background PPI network. Then, DEGs were mapped to the background PPI network to build the sub-network of DEGs which was extended with those proteins (not included in DEGs) interacting with at least three DEGs and trimmed by removing those orphan nodes. The PPI network was visualized using Cytoscape (http://cytoscape.org) (19).

Network topological analysis. The network topological analysis was performed using Network Analyzer (20) plug-in of Cytoscape. Five topological parameters were used in this study, including node degree, average shortest path length (ASPL), closeness centrality (CC), eccentricity (EC) and topological coefficient (TC).

Node degree. In the undirected network, the node degree of a node $x$ is the number of edges linked to $x$. And a node with a high degree is referred to as hub.

Average shortest path length (ASPL). The length of a path between node $x$ and $y$ is the number of edges forming it. The ASPL is the average length of all the shortest paths between nodes in the network, which indicates the expected distance between any two connected nodes.

Closeness centrality $(C C)$. The $\mathrm{CC}$ of a node $x$ is the reciprocal of the ASPL from $x$ to other nodes in the network. The CC of each node is a number between 0 and 1. Closeness centrality is a measure of how quickly information spreads from a given node to other reachable nodes in the network.

Eccentricity $(E C)$. The EC of a node $x$ is the maximum non-infinite length of a shortest path between $\mathrm{n}$ and another node in the network. Specially, the network diameter is the maximum node EC.

Topological coefficient (TC). The TC of a node $x$ is a relative measure for the extent to which a node shares neighbors with other nodes.

Weighting of DEGs based on deviation score and node degree. For each gene, a reference interval (RI) was defined as [min, max] in which min refers to the mean - SD (standard deviation) based on the expression value vector of this gene in NARLC-SCC patients and max refers to the mean + SD. Those genes with an expression value within the RI were thought to resemble the expression pattern of NARLC-SCC patients; otherwise an ARLC-SCC pattern was preferred. Then for gene $x$, the difference beyond the RI in each ARLC-SCC patient was summed up as its deviation score of expression. A higher deviation score represents a larger difference between the expression values of the gene in ARLC-SCC patients and those in NARLC-SCC. Meanwhile, a higher node degree of gene $x$ in the PPI network indicates that it has more neighbors and is of more biological importance. In this study, these two measures were integrated as following to weigh the importance 
of a gene to ARLC-SCC carcinogenesis. The importance score $\mathrm{W}$ of gene $x$ was calculated as below:

$$
\begin{gathered}
\mathrm{W}=\text { score } \mathrm{x} \text { degree } \\
\text { score }=\sqrt{\sum_{i}^{n}\left(d_{i}-d\right)^{2}}
\end{gathered}
$$

in which $d_{i}$ is the expression value of gene $x$ in ARLC-SCC sample $i$, and $n$ is the amount of ARLC-SCC samples. The value of $d$ was set as max if $d_{i}$ was larger than max, otherwise it was set as $\min$. Each score in a sample was scaled into the range of 0 and 10 . The degree was $\log _{2}$ normalized. Finally all genes were sorted based on the value of $W$, and the top $50 \%$ of genes were selected as important genes which were used for further analysis.

Pathway enrichment analysis. To further investigate the functional pathways regulated by these important genes, the Kyoto Encyclopedia of Genes and Genomes (KEGG, http:// www.genome.jp/kegg) pathway (21) enrichment analysis was performed with the important upregulated and downregulated genes, respectively, using online tools from the Database for Annotation, Visualization and Integrated Discovery (DAVID; http://david.abcc.ncifcrf.gov) (22). At the significance cut-off $\mathrm{P}<0.1$ in Fisher exact test, 22 enriched pathways were obtained, among which 12 were kept when the statistical significance was set as $\mathrm{P}<0.05$. To keep sufficient amount of pathways for statistical analysis and subsequent hierarchical clustering, the cut-off of the P-value was set as 0.1 . The pathways enriched by either upregulated or downregulated genes were combined together as important pathways for further analysis.

Hierarchical clustering based on pathway interference. For pathway $\mathrm{p}$ in important pathways, the interference (defined as pathscore) in each tumor sample was assessed as below:

$$
\text { pat hscore }=\log \frac{\sqrt{\sum_{i}^{m} w\left(d_{i}-\dot{d}_{i}\right)^{2}}}{\sqrt{\sum_{j}^{n} w\left(d_{j}-\dot{d}_{j}\right)^{2}}}
$$

Assuming there are $m$ upregulated and $\mathrm{n}$ downregulated genes in pathway $p, d_{i}$ refers to the mean of expression values of the $i$-th upregulated gene in NARLC-SCC samples, while $d_{i}$ means that of the $j$-th downregulated gene. Each gene is weighted with its degree in the network as coefficient $w$. A positive pathscore means this pathway is upregulated in the tumor patients compared with the average level in all NARLC-SCC patients, while a negative pathscore means downregulated. Finally, hierarchical clustering was performed on both samples and pathways using the software Cluster 3.0 (http://bonsai.ims.utokyo.ac.jp/ mdehoon/software/cluster/) (23). Before clustering, the pathscore matrix was log-transformed and median-center scaled. Then, the similarity matrix was calculated using the correlation center. The clustering results were visualized via the Java plugin TreeView (http://jtreeviewsourceforge.net/) (24).

Pathway correlation analysis. The correlation between two pathways was measured with Spearman's rank correlation
Table I. Comparison of network topological parameters in the ARLC-SCC-specific sub-network and the background PPI network.

\begin{tabular}{lcc}
\hline Parameter & $\begin{array}{c}\text { ARLC-SCC-specific } \\
\text { sub-network }\end{array}$ & $\begin{array}{c}\text { Background } \\
\text { PPI network }\end{array}$ \\
\hline Degree & 5.23 & 7.01 \\
EC & 8.52 & 6.51 \\
ASPL & 4.17 & 2.97 \\
CC & 0.24 & 0.35 \\
TC & 0.24 & 0.17 \\
\hline
\end{tabular}

EC, eccentricity; ASPL, average shortest path length; CC, closeness centrality; TC, topological coefficient; ARLC-SCC, asbestos-related squamous cell carcinoma; PPI, protein-protein interaction

coefficient of pathscores across all the samples via software SPSS 19.0 (IBM Corp., Armonk, NY, USA). The pathway pairs with a larger absolute value of $r$ than 0.5 and a significance $\mathrm{P}<0.05$ were assumed to be the significantly correlated pathway pairs. Based on the distribution of DEGs in each pathway, crosstalk genes among these correlated pathways were obtained. The cross-talk genes may influence multiple functional correlated pathways and could be used as critical biomarkers and multi-effect drug targets. Identification of these biomarkers is meaningful for both the understanding of the mechanisms and the clinical diagnosis and therapy for ARLC-SCC.

\section{Results}

Differentially expressed gene extraction. The gene expression difference between 26 ARLC-SCC and 30 NARLC-SCC patients was detected using package LIMMA in Bioconductor, and finally 1,333 DEGs were obtained, in which 391 genes were upregulated and 942 genes were downregulated in the ARLC-SCC patients.

PPI network construction. The background PPI network was constructed based on data from BioGrid and HPRD databases, including 14,553 proteins and 662,360 interactions. To obtain the interaction relationship among these 1,333 DEGs, they were mapped to the background PPI network. Then, the PPI subnetwork of DEGs (Fig. 1) was constructed using Cytoscape, in which 981 nodes and 2,568 interactions were kept.

A gene was represented as $h u b$ in the network if it could interact with multiple DEGs, and thereby this gene could regulate multiple biological processes. To highlight these $h u b$ genes, topological analysis was performed on each node in the network. As shown in Fig. 1, the high-degree nodes (red) were enriched at the center region of the network, while the low-degree nodes (green) were mostly distributed at the outer region.

Topological analysis of the PPI network. To further analyze the specificity of the PPI sub-network of DEGs, five topological parameters were measured for the sub-network and background PPI network, respectively. As shown in Table I, the sub-network of DEGs showed a decreased average node degree (5.23) compared with that (7.01) in the background PPI network. 


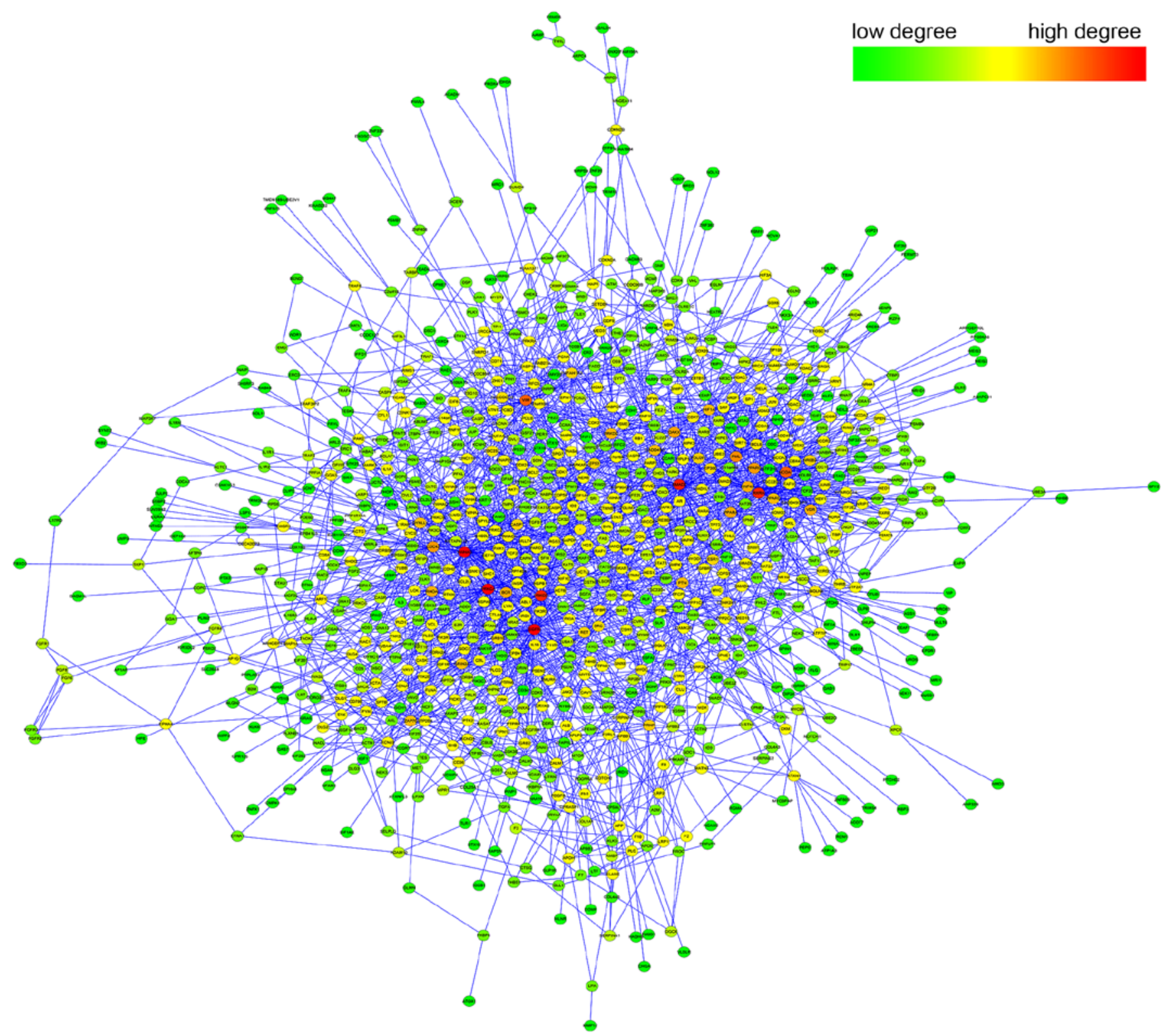

Figure 1. Sub-network of differentially expressed genes in asbestos-related squamous cell carcinoma (ARLC-SCC). Differentially expressed genes (DEGs) were mapped to a background protein-protein interaction (PPI) network and the nodes that were found to interact with at least 3 DEGs were also kept in this sub-network. There are 981 nodes and 2568 interactions in the network. Nodes are colored based on their degrees, among which high-degree nodes are colored red and low-degree nodes are colored green.

Meanwhile, the closeness centrality (CC, 0.24 vs. 0.35$)$ was also reduced in the sub-network. On the contrary, the other three parameters derived from the sub-network were increased compared to those derived from the background network, specifically the average shortest path length (ASPL, 4.17 vs. 2.97) and the eccentricity (EC, 8.52 vs. 6.51 ) were greatly increased and the topological coefficient (TC, 0.24 vs. 0.17 ) with a minor increase. Taken together, these five parameters consistently showed an obvious network efficiency reduction in the sub-network of DEGs compared with that in the background network. The lowered average degree may suggest a reduced contribution of each node to the network, while the increase in ASPL, EC and TC together with the decrease in CC may imply that both the tightness of the network and the information spreading efficiency among the genes were reduced.
Detection of ARLC-SCC-related important genes. To assess the importance of each DEG, an importance score was assigned by integrating its deviation score of expression and node degree in the network. The distribution of these two parameters among all the DEGs is presented as Figs. 2 and 3, respectively. Based on the descending order of importance score, the top 50\% of DEGs were kept as ARLC-SCC-related important genes (524 genes in total). These important genes showed large deviations from the RI and higher node degrees. The higher node degrees indicated that they could interact with multiple DEGs and play a role in multiple biological processes. Thus, these genes are thought to be significantly associated with ARLC-SCC carcinogenesis. Moreover, further studies were carried out on these genes and the biological pathways in which they are involved. 


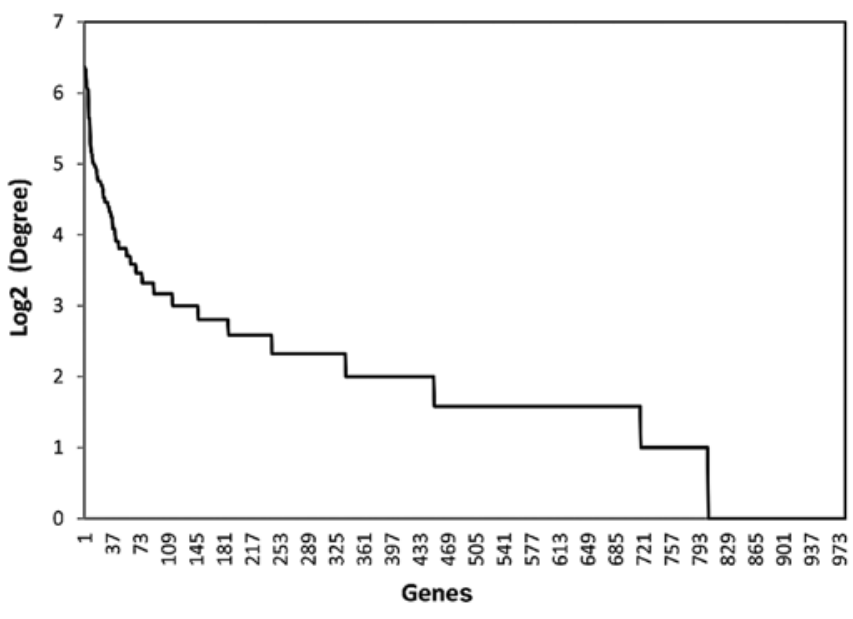

Figure 2. The degree distribution among the 1,333 differentially expressed genes (DEGs). The $\mathrm{x}$-axis represents genes; $\mathrm{y}$-axis represents log-transformed node degree. All the genes are sorted based on descending order of the node degree.

Biological pathways involved in ARLC-SCC carcinogenesis. To get insight into ARLC-SCC-related pathways, the 524 important genes were uploaded to DAVID for KEGG pathway enrichment analysis. Twenty-two KEGG pathways were obtained by combining the enrichment results for the important upregulated and downregulated genes. Specifically, these genes were significantly enriched in specific pathways of cancer, proliferation-related pathways regulating cell cycle and apoptosis, and metastasis-related pathways involved in the regulation of cell adhesion and the cytoskeleton. These results indicated that cancer-related pathways may play an important role in ARLC-SCC development, and the deregulation in cell cycle and apoptosis process may be a major cause for SCC of the lung. Meanwhile, the changes in metastasis-related pathways may account for the poor prognosis and rapid spreading in ARLC-SCC patients.

Hierarchical clustering based on pathway interference pathscore. To measure the extent to which a pathway was interfered with in each tumor patient compared with the average level in all NARLC-SCC patients, a pathscore was calculated for each pathway integrating the deviation score and node degree of all DEGs in the pathway. Then, the pathscore matrix for those 22 pathways in 56 patients was used for hierarchical clustering analysis. The clustering dendrogram was visualized via TreeView and is shown in Fig. 4. As shown in the dendrogram, ARLC-SCC and NARLC-SCC patients were clearly separated based on the pathscore of 22 KEGG pathways, except one NARLC-SCC sample which was grouped into the ARLC-SCC cluster. The clustering analysis in patients indicated a great difference in the expression pattern of these pathways between these two types of SCC samples. On the other side, it also suggests intrinsic associations among these pathways by sharing similar variation trends under different disease phenotypes. For example, the four KEGG pathways such as adherens junction (hsa04520), regulation of actin cytoskeleton (hsa04810), cell cycle (hsa04110) and pathway in cancer (hsa05200) were consistently upregulated in the ARLC-SCC patients compared with the average level of gene

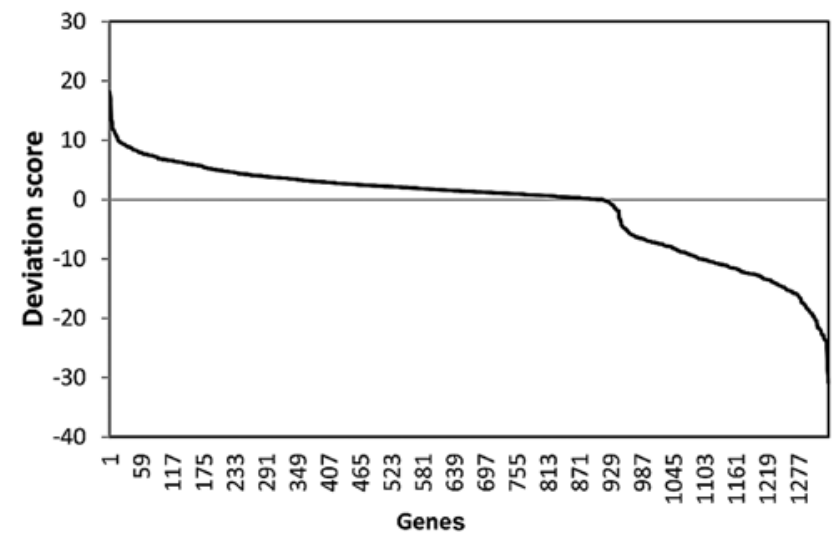

Figure 3. The deviation score distribution among the 1,333 differentially expressed genes (DEGs). The x-axis represents genes; $y$-axis represents deviation score. All the genes are sorted based on descending order of deviation score.

expression in all the NARLC-SCC patients. Thus, identification of the correlated pathways with the same or similar deviation pattern and the cross-talk genes between them are critical for the identification and functional analysis of ARLCSCC-related biomarkers. For this purpose, a correlation study was subsequently applied on the 22 important pathways.

Pathway correlation study and cross-talk gene detection. The correlation between each two important pathways was measured with Spearman's rank correlation coefficient $r$ based on their pathscore across the 56 lung cancer patients. According to the correlation result, 13 significantly correlated pathway pairs were obtained. For better visualization, a pathwaygene bipartite network (Fig. 5) was constructed based on the pathway-gene relationship, gene-gene interaction and pathwaypathway correlation, which included 109 nodes (22 pathways, 87 genes) and 263 edges. As shown in Fig. 5, 12 pathways presented a significant correlation with SNARE interactions in vesicular transport pathway (hsa04130), which indicates that deregulation in the synaptic vesicle transport process may play a central role in the carcinogenesis of ARLC-SCC. Interactions were also found between genes in the same pathway, indicating that these genes interact with each other to regulate the same biological process. In addition, many pathways were not independent but were connected by sharing cross-talk genes. For example, 4 cross-talk genes (EGFR, PRKX, PDGFB and PIK3R3) were shared by 4 pathways such as MAPK signaling pathway, glioma, regulation of actin cytoskeleton and apoptosis. In the same way, the cell cycle, circadian rhythm, oocyte meiosis and neurotrophin signaling pathways were linked together through cross-talk genes [SLK, insulin-like growth factor (IGF1), CDC42 and PRKCA] among which IGF1 and CDC42 codes two critical immunological proteins. This implies that the cross-talk genes between different pathways may take part in the regulation of specific biological functions. The abnormality in these genes may lead to an inbalance of the pathways regulated by them, and finally cause the onset of diseases. Thus, these cross-talk genes could be used as ARLCSCC-specific biomarker genes and even potential multi-effect drug targets. It is possible, that drugs targeting these genes may influence multiple pathways simultaneously 


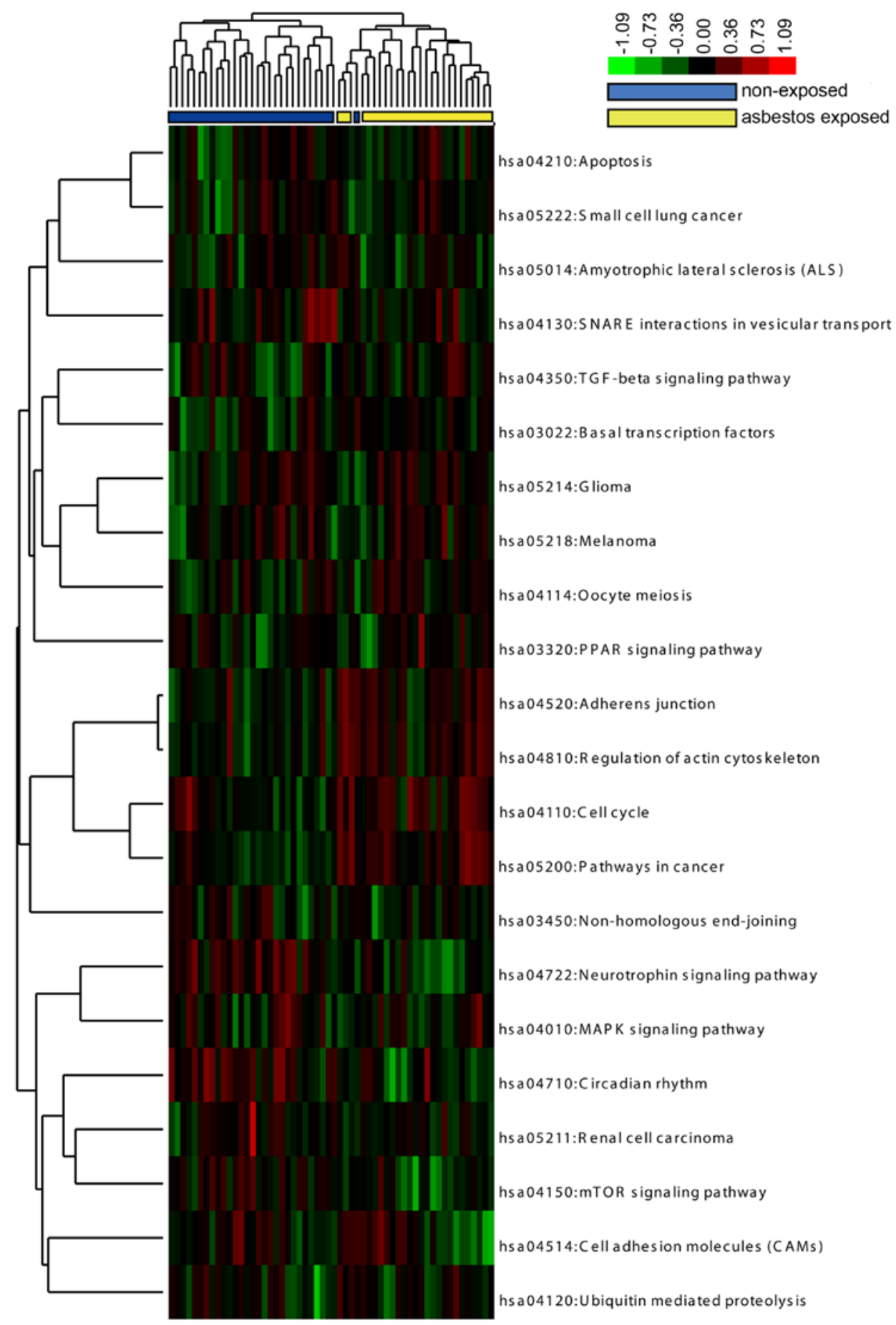

Figure 4. Heat map depicting the pathway interference profile of asbestos-related squamous cell carcinoma (ARLC-SCC) (yellow) and non-asbestos-related squamous cell lung carcinomas (NARLC-SCC) (blue) cases. Pathways were selected on the basis of enrichment statistical significance (P<0.1). Pathways are represented on the $y$-axis and tumor samples are represented on the $\mathrm{x}$-axis. Red areas represent pathways that were upregulated in the tumor samples while green areas represent pathways that were downregulated in the tumor samples.

\section{Discussion}

To date, only one study has attempted to detect the genetic heterogeneity between ARLC-SCC and NARLC-SCC patients. In the study of Wright et al (10), 6 candidate genes (CARD18, MS4A1, ABHD12, API5, ANKRD20A3 and LOC402117) were identified as being differentially expressed between ARLC-SCC and NARLC-SCC cases, while subsequent validations failed to support any of them as markers of asbestos etiology. Compared to their research, a relatively less stringent statistical significance
$(\mathrm{P}<0.05)$ was used to screen the DEGs in the present study. Hence, more candidate genes were reserved for systematical network and pathway analysis, and thereby provided a comprehensive landscape of ARLC-SCC specificity at the gene expression level.

To detect the genetic specificity and biomarker genes in ARLC-SCC patients, gene expression profiles from 26 ARLC-SCC patients and 30 NARLC-SCC patients were systematically analyzed. As a result, 1,333 DEGs were found, for which 524 genes were ARLC-SCC-specific weighted by a novel scoring method. Based on pathway enrichment analysis, 


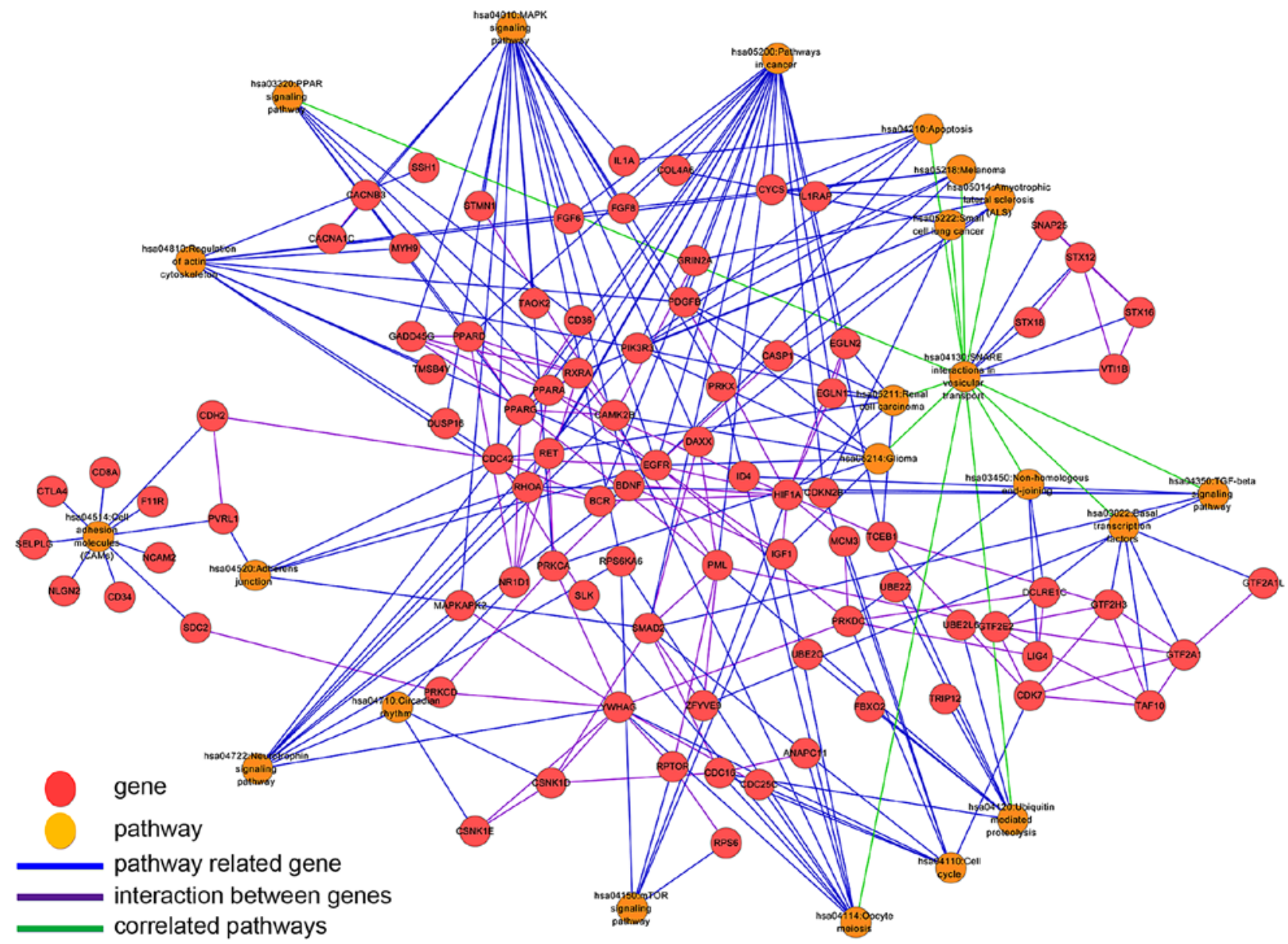

Figure 5. Bipartite interaction network of the pathways and differentially expressed genes (DEGs). In the plot, there are 109 nodes and 263 edges. The nodes colored in orange represent asbestos-related squamous cell carcinoma (ARLC-SCC)-specific pathways, while the nodes in red represent the DEGs in any of these pathways. The affinity of genes and pathways are represented with blue lines and the gene-gene interactions are presented with purple lines. The lines colored in green refer to the pathway-pathway correlations based on the correlation coefficient $(r>0.5$ or $r<-0.5)$ and statistical significance $(\mathrm{P}<0.05)$.

dramatic deviation in 22 KEGG pathways was found between ARLC-SCC and NARLC-SCC patients. Subsequent pathway correlation study showed that functional correlations may exist between these 22 pathways by sharing similar expression deviation patterns or differentially expressed cross-talk genes.

Notably, based on the result of the pathway correlation study, the pathway of SNARE interactions in vesicular transport was found to be significantly correlated with the other 12 enriched pathways of the ARLC-SCC-specific genes, which indicated that abnormal vesicular transport may play a latent role in ARLC-SCC carcinogenesis and development. To date, several transport mechanisms through which cells communicate with the outside environment (within its microenvironment or even at distant sites) have been identified. Among them, vesicular transport, particularly exosome-mediated transport stands out (25). A considerable number of studies over several decades have revealed that the exosome takes part in the transport of multiple biological materials such as proteins, RNAs (26), breakdown product of signaling pathways and viruses (27), and plays a critical role in disease development (28). Furthermore, emerging evidence suggests that exosome-secreted proteins can propel fibroblast growth (29), which may account for the fibrosis of the lungs induced by asbestos exposure in ARLC-SCC cases. To the best of our knowledge, this is the first study to elucidate the potential association between exosome-mediated transport and asbestosis, which may provide a novel direction of drug development for ARLC-SCCs.

Based on the pathway correlation study, the pathways with a similar deviation pattern often presented functional correlation in the 56 SCC patients. These correlated pathways were bridged by the DEGs between them. The shared DEGs constitute cross-talk genes of which the deregulation in expression may simultaneously disturb the function of two or more pathways. Together with PDGFB, PIK3R3 and EGFR, CDC42 is involved in the regulation of the actin cytoskeleton. Abnormal regulation or functioning in cytoskeletal components is often a cause of many diseases including cancers $(30,31)$. Partial disassembly of cytoskeletal actin may account for the respiratory barrier function of pulmonary epithelium induced by ROS in response to asbestos exposure (32). Overexpression of PDGFB can stimulate increased collagen deposition and vascular smooth muscle hyperplasia following asbestos inhalation (33). SLK may promote the cytoskeletal rearrangements and disassembly of actin stress fibers, focal adhesions and induced apoptosis (34), and therefore may contribute to the development of ARLC-SCC. Growth factors such as IGF1 and platelet-derived growth factor (PDGFB) are also known to promote cell cycle re-entry through progression from G1- to S-phase after asbestos exposure (35). Activation of PRKCA is reported to have multiple effects on peribronchiolar cell 
proliferation, pro-inflammatory and pro-fibrotic cytokine expression, and immune cell profiles in the lung, which may lead to asbestos-induced fibrogenesis $(36,37)$. Taken together, the cross-talk genes deregulated in ARLC-SCC patients may be used as biomarkers and multi-effect targets for ARLC-SCC.

Due to the unavailability of these lung cancer patient samples, experimental validations were beyond our reach. Nonetheless, the present study provided a novel insight into the genetic heterogeneity in ARLC-SCC cases. In particular, the finding of the potential role of vesicular transport in ARLC-SCC development provides a new opportunity for the development of a novel therapy for ARLC-SCCs.

In the present study, a novel scoring approach was proposed to detect potential ARLC-SCC-specific marker genes. Based on network topological and pathway correlation analysis, obvious heterogeneity was found between the ARLC-SCC and NARLC-SCC cases. Notably, the undiscovered role of vesicular transport reported herein may provide new insight into the targeted therapy of ARLC-SCCs.

\section{References}

1. Ferlay J, Soerjomataram I, Ervik M, Dikshit R, Eser S, Mathers C, Rebelo M, Parkin DM, Forman D and Bray F: GLOBOCAN 2012 v1.0, Cancer Incidence and Mortality Worldwide: IARC CancerBase No. 11 (Internet). International Agency for Research on Cancer, Lyon, France, 2013. Available from: http://globocan.iarc.fr.2014.

2. Balgkouranidou I, Liloglou T and Lianidou ES: Lung cancer epigenetics: Emerging biomarkers. Biomarkers Med 7: 49-58, 2013.

3. LaDou J: The asbestos cancer epidemic. Environ Health Perspect 112: 285-290, 2004.

4. Nymark P, Guled M, Borze I, Faisal A, Lahti L, Salmenkivi K, Kettunen E, Anttila S and Knuutila S: Integrative analysis of microRNA, mRNA and aCGH data reveals asbestos- and histology-related changes in lung cancer. Genes Chromosomes Cancer 50: 585-597, 2011.

5. Lin RT, Takahashi K, Karjalainen A, Hoshuyama T, Wilson D, Kameda T, Chan CC, Wen CP, Furuya S, Higashi T, et al: Ecological association between asbestos-related diseases and historical asbestos consumption: An international analysis. Lancet 369: 844-849, 2007.

6. Xu X, Li X, Cheng J, Liu Z, Thrall M, Wang X, Wang X and Wong S: Quantitative label-free multimodality nonlinear optical imaging for in situ differentiation of cancerous lesions. Proc. SPIE 8565 (March 8, 2013), Photonic Therapeutics and Diagnostics IX, 85653A, 2013. doi:10.1117/12.2002633; http:// dx.doi.org/10.1117/12.2002633.

7. Henschke CI; International Early Lung Cancer Action Program Investigators: Survival of patients with clinical stage I lung cancer diagnosed by computed tomography screening for lung cancer. Clin Cancer Res 13: 4949-4950, 2007.

8. Henschke CI, Yankelevitz DF, Libby DM, Pasmantier MW, Smith JP and Miettinen OS; International Early Lung Cancer Action Program Investigators: Survival of patients with stage I lung cancer detected on CT screening. N Engl J Med 355: 1763-1771, 2006.

9. Schwartz AG, Prysak GM, Bock $\mathrm{CH}$ and Cote ML: The molecular epidemiology of lung cancer. Carcinogenesis 28: 507-518, 2007.

10. Wright CM, Savarimuthu Francis SM, Tan ME, Martins MU, Winterford C, Davidson MR, Duhig EE, Clarke BE, Hayward NK, Yang IA, et al: MS4A1 dysregulation in asbestos-related lung squamous cell carcinoma is due to CD20 stromal lymphocyte expression. PLoS One 7: e34943, 2012.

11. Wikman H, Ruosaari S, Nymark P, Sarhadi VK, Saharinen J, Vanhala E, Karjalainen A, Hollmén J, Knuutila S and Anttila S: Gene expression and copy number profiling suggests the importance of allelic imbalance in 19p in asbestos-associated lung cancer. Oncogene 26: 4730-4737, 2007.

12. Nymark P, Lindholm PM, Korpela MV, Lahti L, Ruosaari S, Kaski S, Hollmén J, Anttila S, Kinnula VL and Knuutila S: Gene expression profiles in asbestos-exposed epithelial and mesothelial lung cell lines. BMC Genomics 8: 62, 2007.
13. Nymark P, Wikman H, Hienonen-Kempas T and Anttila S: Molecular and genetic changes in asbestos-related lung cancer. Cancer Lett 265: 1-15, 2008.

14. Ruosaari S, Hienonen-Kempas T, Puustinen A, Sarhadi VK, Hollmén J, Knuutila S, Saharinen J, Wikman H and Anttila S: Pathways affected by asbestos exposure in normal and tumour tissue of lung cancer patients. BMC Med Genomics 1: 55, 2008.

15. Wright CM, Larsen JE, Hay ward NK, Martins MU, Tan ME, Davidson MR, Savarimuthu SM, McLachlan RE, Passmore LH, Windsor MN, et al: ADAM28: A potential oncogene involved in asbestos-related lung adenocarcinomas. Genes Chromosomes Cancer 49: 688-698, 2010.

16. Smyth GK: Limma: Linear models for microarray data. In: Bioinformatics and Computational Biology Solutions Using $\mathrm{R}$ and Bioconductor. Springer, pp397-420, 2005. http://link. springer.com/chapter/10.1007\%2F0-387-29362-0_23\#page-1.

17. Chatr-Aryamontri A, Breitkreutz B-J, Heinicke S, Boucher L, Winter A, Stark C, Nixon J, Ramage L, Kolas N, O'Donnell L, et al: The BioGRID interaction database: 2013 update. Nucleic Acids Res 41: D816-D823, 2013.

18. Keshava Prasad TS, Goel R, Kandasamy K, Keerthikumar S, Kumar S, Mathivanan S, Telikicherla D, Raju R, Shafreen B, Venugopal A, et al: Human protein reference database-2009 update. Nucleic Acids Res 37: D767-D772, 2009.

19. Saito R, Smoot ME, Ono K, Ruscheinski J, Wang PL, Lotia S, Pico AR, Bader GD and Ideker T: A travel guide to Cytoscape plugins. Nat Methods 9: 1069-1076, 2012.

20. Doncheva NT, Assenov Y, Domingues FS and Albrecht M: Topological analysis and interactive visualization of biological networks and protein structures. Nat Protoc 7: 670-685, 2012.

21. Kanehisa M, Goto S, Sato Y, Kawashima M, Furumichi M and Tanabe M: Data, information, knowledge and principle: Back to metabolism in KEGG. Nucleic Acids Res 42: D199-D205, 2014.

22. Huang W, Sherman BT and Lempicki RA: Systematic and integrative analysis of large gene lists using DAVID bioinformatics resources. Nat Protoc 4: 44-57, 2009.

23. de Hoon MJ, Imoto S, Nolan J and Miyano S: Open source clustering software. Bioinformatics 20: 1453-1454, 2004.

24. Saldanha AJ: Java Treeview - extensible visualization of microarray data. Bioinformatics 20: 3246-3248, 2004.

25. EL Andaloussi S, Mäger I, Breakefield XO and Wood MJ: Extracellular vesicles: Biology and emerging therapeutic opportunities. Nat Rev Drug Discov 12: 347-357, 2013.

26. Vinciguerra $P$ and Stutz F: mRNA export: An assembly line from genes to nuclear pores. Curr Opin Cell Biol 16: 285-292, 2004.

27. Schorey JS and Bhatnagar S: Exosome function: From tumor immunology to pathogen biology. Traffic 9: 871-881, 2008.

28. Staals RH and Pruijn GJ: The human exosome and disease. In: RNA Exosome. Springer, pp132-142, 2010. http://link.springer. com/chapter/10.1007\%2F978-1-4419-7841-7_11\#page-1.

29. Azmi AS, Bao B and Sarkar FH: Exosomes in cancer development, metastasis, and drug resistance: A comprehensive review. Cancer Metastasis Rev 32: 623-642, 2013.

30. Reymond N, Im JH, Garg R, Vega FM, Borda d'Agua B, Riou P, Cox S, Valderrama F, Muschel RJ and Ridley AJ: Cdc 42 promotes transendothelial migration of cancer cells through $\beta 1$ integrin. J Cell Biol 199: 653-668, 2012.

31. Lee SH and Dominguez R: Regulation of actin cytoskeleton dynamics in cells. Mol Cells 29: 311-325, 2010.

32. Boardman KC, Aryal AM, Miller WM and Waters CM: Actin re-distribution in response to hydrogen peroxide in airway epithelial cells. J Cell Physiol 199: 57-66, 2004.

33. Li J, Poovey HG, Rodriguez JF, Brody A and Hoyle GW: Effect of platelet-derived growth factor on the development and persistence of asbestos-induced fibroproliferative lung disease. J Environ Pathol Toxicol Oncol 23: 253-266, 2004.

34. Sabourin LA, Tamai K, Seale P, Wagner J and Rudnicki MA: Caspase 3 cleavage of the Ste20-related kinase SLK releases and activates an apoptosis-inducing kinase domain and an actindisassembling region. Mol Cell Biol 20: 684-696, 2000.

35. Brody AR: Asbestos-induced lung disease. Environ Health Perspect 100: 21-30, 1993.

36. Shukla A, Lounsbury KM, Barrett TF, Gell J, Rincon M, Butnor KJ, Taatjes DJ, Davis GS, Vacek P, Nakayama KI, et al: Asbestos-induced peribronchiolar cell proliferation and cytokine production are attenuated in lungs of protein kinase $\mathrm{C}-\delta$ knockout mice. Am J Pathol 170: 140-151, 2007.

37. Shukla A, Barrett TF, Nakayama KI, Nakayama K, Mossman BT and Lounsbury KM: Transcriptional upregulation of MMP12 and MMP13 by asbestos occurs via a PKCdelta-dependent pathway in murine lung. FASEB J 20: 997-999, 2006. 